\title{
Treatment of Invasive Fungal Infections: Stability of Voriconazole Infusion Solutions in PVC Bags
}

Andréa I.H. Adams, Lucia N. Morimoto, Leonardo Z. Meneghini and Ana M. Bergold

Pharmacy School of Federal University of Rio Grande do Sul (UFRGS); Porto Alegre, RS, Brazil

\begin{abstract}
Voriconazole is a novel broad-spectrum antifungal drug, employed in the treatment of invasive fungal infections, and represents an alternative to amphotericin $B$ treatment. The manufacturer recommends that any unused reconstituted product should be stored at $2^{\circ} \mathrm{C}$ to $8^{\circ} \mathrm{C}$, for no more than $24 \mathrm{~h}$, but no recommendations about i.v. infusion solutions are given. Previous works have reported on the stability of voriconazole in polyolefin bags and

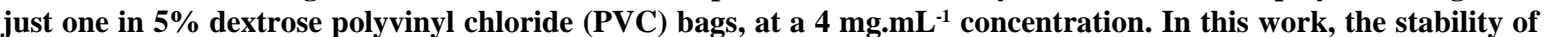
voriconazole as an i.v. infusion solution in $0.9 \%$ sodium chloride and in $5 \%$ dextrose, in PVC bags, at $0.5 \mathrm{mg}^{\mathrm{mL}} \mathrm{m}^{-1}$, stored at $4{ }^{\circ} \mathrm{C}$ and at room temperature, protected from light, was evaluated. These infusion solutions were analyzed for a 21-day period. Chemical stability was evaluated by HPLC assay. Visual inspection was performed and pH of the solutions was measured. No color change or precipitation in the solutions was observed. The drug content remained above $\mathbf{9 0 \%}$ for 11 days in $\mathbf{0 . 9 \%}$ sodium chloride and for 9 days in $\mathbf{5 \%}$ dextrose solutions. The i.v. infusion solutions stored at room temperature were not stable. At room temperature, the voriconazole content dropped down to 88.3 and $\mathbf{8 6 . 6 \%}$, in $\mathbf{0 . 9 \%}$ sodium chloride or $5 \%$ dextrose solutions, respectively, two days after admixture. Assays performed at the end of the study suggest the sorption of voriconazole by the PVC bags. The results of this study allow cost-effective batch production in the hospital pharmacy.
\end{abstract}

Key-Words: Antifungal drugs, voriconazole, voriconazole stability, voriconazole infusion solutions, PVC bags.

The past two decades have witnessed an increase in the incidence of nosocomial fungal infections. Fungal sepsis has increased by more than $200 \%$ in this period [1] and represents a major problem, given the relatively poor response rates, especially for aspergillosis, and the high cost of the therapies [2]. The increase in the incidence of fungal infections may be attributed primarily to increased numbers of critically ill and immunocompromised patients, including those with AIDS, cancer patients undergoing chemotherapy, and organ transplant recipients taking immunosuppressive drugs. Other possible reasons for the increased incidence of fungal infections include the growing number of patients undergoing invasive medical procedures, receiving broad-spectrum antibiotics or long-term corticosteroid therapy and receiving parenteral nutrition or hemodialysis [3].

Candida is responsible for ca. $80 \%$ of nosocomial fungal infections, with Candida albicans being the predominant Candida pathogen [4]. Aspergillus spp. are less common, causing ca. $4 \%$ to $5 \%$ of nosocomial fungal infections [3]. These pathogens are related to an extremely high mortality rate: around $40 \%$ to $60 \%$ for Candida [5] and $85 \%$ for Aspergillus [3], even with aggressive antifungal therapy. Although Candida and Aspergillus species still represent the vast majority of fungal isolates encountered in human pathology, a number of new species - both yeasts and filamentous fungi - are increasingly recognized as opportunistic pathogens [6,7].

Received on 10 April 2008; revised 18 September 2008.

Address for correspondence: Dr. Ana M. Bergold. Faculdade de Farmácia - Universidade Federal do Rio Grande do Sul. Av. Ipiranga, 2752. CEP 90610-000. Porto Alegre, RS/Brasil. ana.bergold@ufrgs.br.
The number of available antifungal agents suitable for treating systemic fungal infections is limited. Until recently, the arsenal that was available consisted mainly of the polyene antibiotic amphotericin B, some azole derivatives, the allylamines-thiocarbamates and 5-flucytosine. In the past decade, however, new compounds were licensed, including azole compounds and the candins [7]. Voriconazole (Figure 1) is an azole compound, structurally related to fluconazole, where one of the triazole moieties was replaced by a fluoropyrimidine group and an alpha methyl group was introduced. These modifications resulted in an enhanced spectrum of antifungal activity and in increased in vitro potency [8].

Amphotericin B deoxycholate and voriconazole are the only two antifungal medications currently approved as primary therapy for invasive aspergillosis infection in the U.S.. Second line treatments include lipid-based formulations of amphotericin, itraconazole and caspofungin $[9,10]$.

Figure 1. Chemical structure of voriconazole.

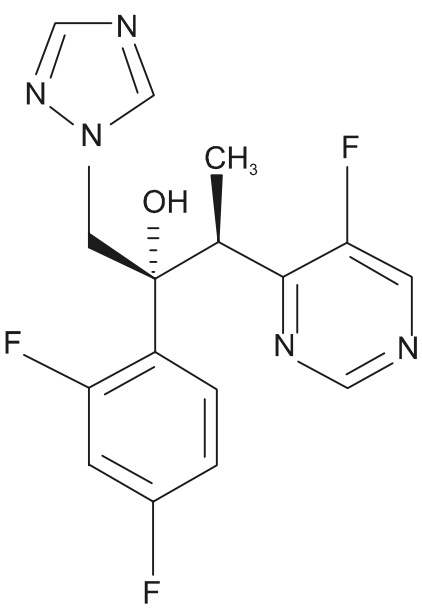


Voriconazole was authorized for use by the FDA in the U.S. and by ANVISA in Brazil in 2002, for the treatment of invasive fungal infections [6]. It is especially recommended for the treatment of invasive aspergillosis and of infections caused by Scedosporium, Fusarium spp and resistant Candida species [11].

Voriconazole is available as an injection, tablets and oral suspension. The i.v. product is supplied in a single-use vial containing $200 \mathrm{mg}$ of voriconazole and 3,200 mg of sulfobutyl ether beta-cyclodextrin sodium, which makes the drug soluble and allows its parenteral administration. The powder must be reconstituted with sterile water for injection and this solution must be diluted to a final concentration of 0.5 to $5 \mathrm{mg} / \mathrm{mL}$ with suitable diluents, such as $0.9 \%$ sodium chloride or $5 \%$ dextrose [12].

The manufacturer recommends that any unused reconstituted solution should be discarded after $24 \mathrm{~h}$, since it has been demonstrated to be stable for this period, stored at 2 to $8^{\circ} \mathrm{C}$. There are no recommendations about the stability of the infusion solutions or about their compatibility with different polymers of infusion bags [12]. In relation to the stability of infusion solutions, it was reported that $2 \mathrm{mg} \cdot \mathrm{mL}^{-1}$ voriconazole in $0.9 \%$ sodium chloride infusion bags is stable at $2-8{ }^{\circ} \mathrm{C}$ for 32 days; the same shelf life was verified for the reconstituted product [13]. It was also reported that $2 \mathrm{mg} . \mathrm{mL}^{-1}$ voriconazole, protected from light, was stable for eight days when stored at 4 or $25^{\circ} \mathrm{C}$ diluted in $0.9 \%$ sodium chloride solution, and for six days at $4{ }^{\circ} \mathrm{C}$ and four days at $25^{\circ} \mathrm{C}$ in $5 \%$ dextrose solution [14]. In both studies, voriconazole was stored in multilayer polyolefin bags. The stability of voriconazole was studied in PVC bags, at 4 mg.mL $\mathrm{m}^{-1}$ in 5\% dextrose, stored at $4{ }^{\circ} \mathrm{C}$. Under these conditions, voriconazole solutions are stable for 15 days [15].

The role voriconazole plays in the treatment of fungal infections, the high cost of the therapy, and the dose adjustments which produce residual product justify more studies on its stability. The aim of this work was to study the stability of voriconazole as i.v. infusion solutions in PVC bags, in a diluent other than dextrose, since for some patients this diluent is not recommended. The chemical stability of voriconazole was determined as infusion solutions in $0.9 \%$ sodium chloride and 5\% dextrose, stored in PVC bags over 21 days, maintained at $4-7$ and $19-24{ }^{\circ} \mathrm{C}$, at $0.5 \mathrm{mg}^{\mathrm{mL}} \mathrm{m}^{-1}$. This concentration represents the lowest concentration recommended for an i.v. infusion solution.

\section{Material and Methods}

Reagents and Drugs

Vials of lyophilized voriconazole powder (Vfend $₫ 200 \mathrm{mg}$, Pfizer, lot 5163904) were reconstituted with $19 \mathrm{~mL}$ of sterile water for injection to yield $20 \mathrm{~mL}$ of $10 \mathrm{mg} \cdot \mathrm{mL}^{-1}$ solution. PVC bags containing $0.9 \%$ sodium chloride injection $(50 \mathrm{~mL}$, Halex Istar, lot IB 607/05) and 5\% dextrose injection (100 mL, Halex Istar, lot IB 631/03) were used. Voriconazole standard, with an assigned purity of $99.75 \%$ was provided by Gencor Pacific
Limited, Hong Kong. For high-performance liquid chromatography assay, methanol (HPLC grade, Merck, Germany), triethylamine and orthophosphoric acid reagent grade (Merck, Germany), and distilled water purified by a MilliQ Plus water system (Millipore, USA) were used.

\section{Chromatographic Apparatus and Conditions}

A Shimadzu HPLC system (Kyoto, Japan) provided with a LC-10AD ${ }_{\mathrm{vp}}$ pump, a Rheodyne ${ }^{\circledR}$ manual injector (model 7725i), a SPD-M10A $A_{\mathrm{vP}}$ diode array detector, DGU-14A degas and an SCL-10A ${ }_{\mathrm{VP}}$ controller with CLASS-VP software was employed. The chromatographic analyses were performed on a Merck LiChrospher ${ }^{\circledR} 100$ RP-8 (125 x 4.6 mm I.D., 5 mm particle size) column. The mobile phase was composed of methanol/ $0.6 \%$ triethylamine, $\mathrm{pH} 6.0(50: 50 \mathrm{v} / \mathrm{v})$, at a flow rate of $1 \mathrm{~mL} \cdot \mathrm{min}^{-1}$, and the injection volume was $20 \mu \mathrm{L}$. UV detection was performed at $255 \mathrm{~nm}$ and the identity of peaks was determined by comparing the elution times and ultraviolet spectra of samples and standard solution, supplied by the PDA detector.

\section{HPLC Analysis}

Voriconazole concentrations were determined by a stability-indicating HPLC method previously described in the literature [16], employing exactly the same conditions that are described in the Chromatographic apparatus and conditions section. Chromatographic parameters which allow the adjustment of system performance, were monitored.

The applied method was linear in the range of 0.02 to 0.1 mg.mL ${ }^{-1}$, with a coefficient of correlation of 0.9999 and a linear regression equation of $y=-923.76+27569.82 x$. The method was examined for specificity by carrying out forced degradation studies which demonstrated that voriconazole is sensitive to alkali, acid and oxidizing media, where degradation in alkaline conditions is faster and more intense. In all the conditions employed, peaks of degradation products, when observed (retention times: 2.6 to $7.7 \mathrm{~min}$ ) were resolved from the voriconazole peak (retention time: $8.4 \mathrm{~min}$ ) [16].

In this study, specificity and precision of the assay were evaluated again. The specificity was assessed by the peak purity of all voriconazole peaks, given by the PDA detector. To be considered pure, the peak purity index should be higher than 0.99 [17]. The precision of the method was evaluated by the intra-day relative standard deviation values (RSD) between all the duplicate samples assayed, where RSD values $\leq 2.0 \%$ were considered acceptable.

Stability Study

For the preparation of the i.v. infusion solutions, $2.5 \mathrm{~mL}$ of freshly prepared reconstituted product were introduced into 50 $\mathrm{mL}$ of $0.9 \%$ sodium chloride or $100 \mathrm{~mL}$ of $5 \%$ dextrose PVC bags, from which $2.5 \mathrm{~mL}$ and $52.5 \mathrm{~mL}$ were previously withdrawn, respectively, to obtain a voriconazole concentration of 0.5 mg.mL ${ }^{-1}$. The bags were then shaken to ensure thorough mixing. Three bags of voriconazole were prepared for each of the infusion solutions and temperatures assayed. 
The i.v. infusion solutions were stored under refrigeration $\left(4-7^{\circ} \mathrm{C}\right)$ or at room temperature $\left(19-24^{\circ} \mathrm{C}\right)$, in the dark. They were analyzed at $0,2,4,7,9,11,14$ and 21 days. Before the analysis was conducted, the bags were allowed to reach room temperature and mixed well before sample collection. One milliliter was taken from each of the three bags and diluted with water to $0.05 \mathrm{mg} \cdot \mathrm{mL}^{-1}$, and each bag was assayed in duplicate. Since the method was linear in the range of 0.02 to $0.1 \mathrm{mg} \cdot \mathrm{mL}^{-1}$ [16], even if degradation occurred, the solution concentrations remained in the linear range of the analytical method. The voriconazole reconstitution, the preparation of the i.v. infusion solutions and the sampling at each time were performed using aseptic technique.

The quantification was performed comparing the mean areas of the standard solutions at a concentration of 0.05 mg. $\mathrm{mL}^{-1}$ with the sample solutions. The initial concentrations (day 0 ) were defined as $100 \%$ and subsequent concentrations were expressed as percentages of the initial concentration. The results were reported as means \pm S.D. of the percentages of the initial concentration. The drug solutions were defined as stable if more than $90 \%$ of the initial concentration was maintained. The $\mathrm{pH}$ was measured with a $\mathrm{pH}$ meter at the beginning and at the end of the study (DM 20-Digimed). The appearance and color were accessed by observing samples against black and white backgrounds.

At the end of the study, the bags were totally emptied and washed several times with water, where the last portion of the washing solutions was saved. A-20 mL volume of methanol was added to the bags, which were maintained in a horizontal position and allowed to be in contact with the solvent for 5 days. After this period, an aliquot of the methanol solution and the final washing solution were analyzed by HPLC assay.

\section{Results and Discussion}

Considering the clinical relevance of voriconazole in the treatment of invasive fungal infections, the high cost of this therapy and the lack of studies about its stability, this study was carried out to determine drug stability in the diluents and storage conditions usually employed in Brazilian hospitals. Voriconazole powder for i.v. infusion solutions must be first reconstituted with sterile water for injection and then diluted with appropriate diluents. There are no recommendations with regard to the stability of the infusion solution or about its compatibility with different polymers of infusion bags in the package insert [12]. Two studies reported in the literature employed polyolefin bags (PVC-free) $[13,14]$ and another one used PVC bags [15]. The latter reported voriconazole stability in $5 \%$ dextrose solution, a diluent not recommended for some patients. In the present study, drug stability in PVC bags containing $0.9 \%$ sodium chloride injection was also studied. The cost of polyolefin bags is twice that of PVC bags. In Europe, around $60 \%$ of the bags used are made of PVC, and in the U.S., around 80\%. In Brazil, closed system PVC bags are being introduced, which will reduce the most important problem related to the open system bags currently used: the risk of contamination.

The degradation of voriconazole was determined by a stability indicating method, previously validated [16]. Moreover, chromatographic parameters and specificity of the assay were monitored. The chromatographic parameters determined indicate that the conditions tested provided a wellbehaved chromatographic system, essential for accurate and precise results. The following values were obtained: theoretical plates $\geq 2,500$, tailing factor $\leq 1.6$ and capacity factor $\leq 7.6$, all of which are in accordance with established values [18]. The specificity of the assay was evaluated again in this experiment, employing a PDA detector. To be considered specific, which is an essential characteristic for stability studies, an assay method should demonstrate that it is able to separate and quantify the drug from a physical mixture of the drug, degradation products and excipients. As mentioned in the HPLC analysis section, the voriconazole peak was resolved from the peaks of the degradation products, obtained in the forced degradation studies [16]. Besides, the peak purity index was determined by the PDA detector, the most popular technique used to assay it. The purity of the peak attributed to voriconazole was higher than 0.9999 in all assays, showing that no impurities or degradation products or excipients coeluted with the parent drug, indicating the specificity of the analytical method [17]. The precision of the assay was

Table 1. Stability of $0.5 \mathrm{mg} / \mathrm{mL}$ voriconazole in $0.9 \%$ sodium chloride and $5 \%$ dextrose at $4-7$ and $19-24{ }^{\circ} \mathrm{C}$.

\begin{tabular}{|c|c|c|c|c|c|c|c|c|}
\hline \multirow[b]{2}{*}{$\begin{array}{l}\text { Diluent and } \\
\text { storage temperature }\end{array}$} & \multirow[b]{2}{*}{$\begin{array}{l}\text { Initial } \\
\text { concentration } \\
(\mathbf{m g} / \mathbf{m L})\end{array}$} & \multicolumn{6}{|c|}{$\%$ initial drug concentration remaining $\pm \mathbf{S D}^{\mathbf{a}}$} & \multirow[b]{2}{*}{ Day 21} \\
\hline & & Day 2 & Day 4 & Day 7 & Day 9 & Day 11 & Day 14 & \\
\hline \multicolumn{9}{|l|}{ 0.9\% sodium chloride } \\
\hline $4-7^{\circ} \mathrm{C}$ & $0.52 \pm 0.03$ & $92.3 \pm 1.2$ & $91.7 \pm 1.2$ & $91.7 \pm 0.6$ & $91.2 \pm 1.9$ & $91.5 \pm 1.2$ & $89.1 \pm 1.0$ & $87.3 \pm 0.6$ \\
\hline $19-24^{\circ} \mathrm{C}$ & $0.47 \pm 0.02$ & $88.3 \pm 1.3$ & $83.2 \pm 1.1$ & $76.4 \pm 0.7$ & $75.2 \pm 1.3$ & $76.0 \pm 1.3$ & $71.6 \pm 0.8$ & $63.6 \pm 2.4$ \\
\hline \multicolumn{9}{|l|}{$5 \%$ dextrose } \\
\hline $4-7^{\circ} \mathrm{C}$ & $0.50 \pm 0.01$ & $92.4 \pm 0.9$ & $93.6 \pm 1.0$ & $92.2 \pm 0.7$ & $92.9 \pm 1.2$ & $87.7 \pm 1.9$ & $89.2 \pm 2.5$ & $88.0 \pm 0.4$ \\
\hline $19-24^{\circ} \mathrm{C}$ & $0.51 \pm 0.01$ & $86.6 \pm 0.7$ & $87.9 \pm 2.1$ & $82.4 \pm 0.9$ & $80.9 \pm 3.1$ & $75.9 \pm 1.3$ & $75.5 \pm 0.5$ & $69.7 \pm 2.3$ \\
\hline
\end{tabular}

a Standard deviation. 
confirmed by intra-day relative standard deviation (RSD) values less than $2.0 \%$.

No color changes, haziness or precipitation were observed in the infusion solutions until the end of the study. The $\mathrm{pH}$ values decreased slightly during the period of analysis: from $5.93 \pm 0.09$ to $5.80 \pm 0.10$ and from $5.90 \pm 0.12$ to $5.60 \pm 0.08$, for $0.9 \%$ sodium chloride solutions, maintained at $4{ }^{\circ} \mathrm{C}$ or at room temperature, respectively. Similar results were observed for the dextrose solutions, which showed a decrease from $4.42 \pm$ 0.10 to $4.30 \pm 0.13$ and from $4.29 \pm 0.09$ to $4.15 \pm 0.06$, stored at $4{ }^{\circ} \mathrm{C}$ or at room temperature, respectively.

Table 1 presents the results obtained in the stability test. The percentages of the initial concentration indicate that the compound was stable in $0.9 \%$ sodium chloride for 11 days and for 9 days in $5 \%$ dextrose solutions, stored at $4{ }^{\circ} \mathrm{C}$ in PVC bags, protected from light. The infusion solutions maintained under refrigeration were more stable than those maintained at room temperature, an effect observed in both diluents. At room temperature, the drug content percentages dropped to 88.3 and 86.6 , in $0.9 \%$ sodium chloride and 5\% dextrose solutions, respectively, only two days after preparation. The faster decrease of drug content in the bags stored at room temperature was also observed by Sahraoui et al. (2006) who studied the stability of voriconazole in polyolefin bags [14].

No degradation products were formed in the samples stored at $4^{\circ} \mathrm{C}$ or room temperature, which can be observed in chromatograms obtained at day 21 (Figure 2). This result suggests that the degradation products do not have a chromophore group, that voriconazole was decomposed to low molecular weight fractions or that sorption of voriconazole by the PVC bags occurred. In order to confirm the latter possibility, the PVC bags were totally emptied, washed with water, where the last portion of washing solution was saved. Methanol was introduced and kept inside the bags for 5 days,

Figure 2. Chromatograms of voriconazole infusion solution in $0.9 \%$ sodium chloride (A) and in $5 \%$ dextrose (B) at zero time and day 21 after admixture, maintained at $4-7^{\circ} \mathrm{C}$.

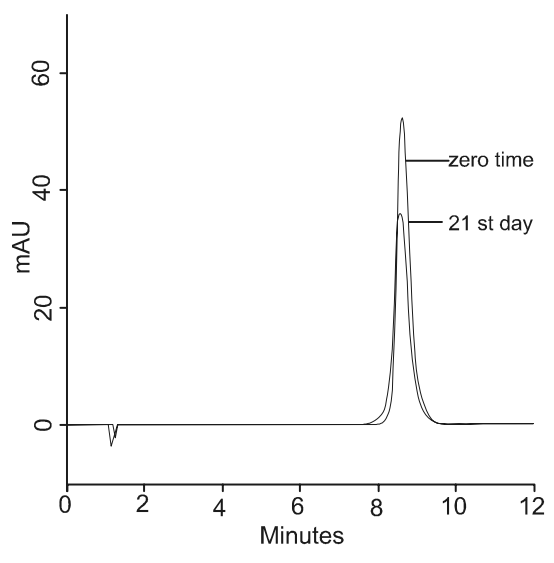

A

Figure 3. Chromatograms of voriconazole, extracted with methanol from PVC bags that previously contained voriconazole in $0.9 \%$ sodium chloride, after 5-day period of contact (A); last washing aqueous solutions (B).

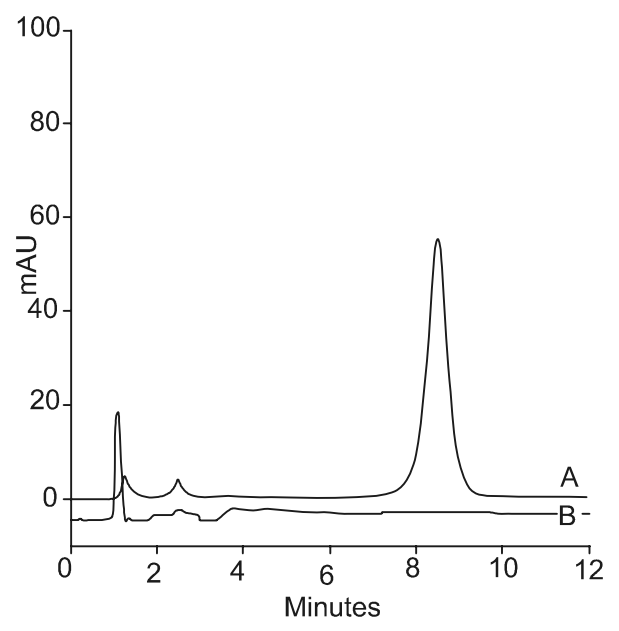

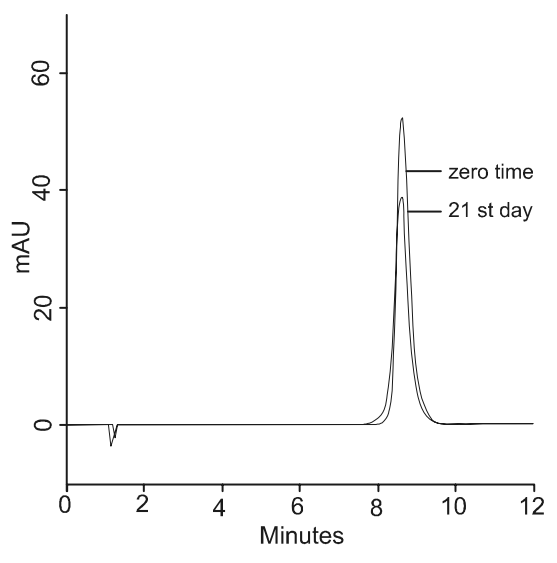

B

Figure 4. Chromatograms of voriconazole, extracted with methanol from PVC bags that previously contained voriconazole in 5\% dextrose, after 5-day period of contact (A); last washing aqueous solutions (B).

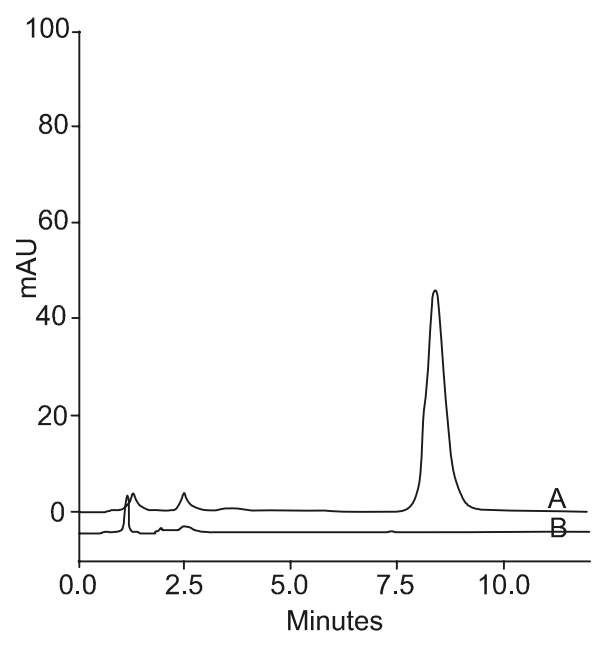


in order to extract any bound drug from the bags. Voriconazole was detected in the methanol solutions (Figures 3A and 4A), but not in the washing solutions (Figures $3 \mathrm{~B}$ and $4 \mathrm{~B}$ ). This fact suggests that the amount in the methanol solutions came exclusively from adsorbed and/or absorbed drug. As noted in previous works, the $\mathrm{pH}$ of the solution is an essential factor that influences the sorption of drugs by PVC bags. The nonionized drug species are more lipophilic and thus, more favorably absorbed by the plastic [19-22]. The relative amount of non-ionized form depends on the solution $\mathrm{pH}$ and on the drug pKa. Voriconazole is a weak base (pKa 1.76) [23] and in the range of $\mathrm{pH}$ values obtained at the end of the study (5.93 to 4.15 ), the percentage of non-ionized drug form is higher than $99.5 \%$. A faster decrease in $\mathrm{pH}$ was observed in infusion solutions stored at room temperature. As previously reported [21], higher temperatures can increase the solute diffusion rate through the plastic matrix, increasing drug binding.

Analysis made by PDA detector at day 21 showed no other peaks in the visible and UV range and the purity of voriconazole peak, which reinforces the notion of drug sorption by the bags.

The result obtained here differs, in part, from that obtained by Cadrobbi et al. [16]. They found that voriconazole in 5\% dextrose solution, stored at $4^{\circ} \mathrm{C}$, at a concentration of $4 \mathrm{mg} \cdot \mathrm{mL}^{-1}$, was stable for a 15-day period, while we found a 9-day stability period. This difference can be an effect of the voriconazole concentration, since we used the lowest concentration recommended by the manufacturer, $0.5 \mathrm{mg} \cdot \mathrm{mL}^{-1}$. Moorhatch and Chiou [19] reported different sorption effects with PVC bags depending ob drug concentration. For vitamin A and methohexital, the bound percentage is not influenced by concentration. On the other hand, an increase in warfarin concentration results in a decrease in the bound percentage. Thus, in the last case, more dilute solutions lost a higher percent of total drug to the plastic, as observed in the present study. However, other studies showed no correlation between drug sorption and concentration [20,21]. Therefore, the question about concentration effect posed here should be examined by additional studies, employing other voriconazole concentrations.

\section{Conclusion}

The present study shows that voriconazole as an infusion solution in PVC bags, at $0.5 \mathrm{mg} \cdot \mathrm{mL}^{-1}$, stored at $4-7^{\circ} \mathrm{C}$ and protected from light, is stable for 11 days in $0.9 \%$ sodium chloride and for 9 days in 5\% dextrose solutions. At room temperature, stored in PVC bags and protected from light, i.v. solutions are stable for less than 2 days, suggesting that they should be administered immediately after their preparation. These results allow a reduction of costs by batch production at the hospital pharmacy. Considering that there is no preservative in voriconazole injection, the reconstitution and i.v. admixtures should be carried out in aseptic areas, in order to avoid microbial contamination and to allow its use for the suggested periods, for which its chemical stability was determined.

\section{Acknowledgements}

The authors wish to thank CNPq for the research grants, Cristalia Laboratories for the supply of voriconazole reference substance, Farmoterápica for the donation of PVC bags and São Vicente de Paulo Hospital for the supply of voriconazole injection.

\section{References}

1. Martin G.S., Mannino D.M., Eaton S., et al. The epidemiology of sepsis in the United States from 1979 through 2000. N Engl J Med 2003;348:1546-54.

2. Klastersky J. Empirical antifungal therapy. Int J Antimicrob Agents 2004;23:105-12.

3. Stone E.A., Fung H.B., Kirschenbaum H.L. Caspofungin: an echinocandin antifungal agent. Clin Ther 2002;24:351-77.

4. Wilson L., Reyes C.M., Stolpman M., et al. The direct cost and incidence of systemic fungal infections. Value Health 2002;5:26-34.

5. Colombo A.L., Guimarães T. Epidemiologia das infecções hematogênicas por Candida spp. Rev Soc Bras Med Trop 2003;36:599-607.

6. Moretti M.L. A importância crescente das infecções fúngicas. Rev Panam Infect 2007;9.

7. Maertens, J.A. History of the development of azole derivatives. Clin Microbiol Infect 2004;10:1-10.

8. Fromtling R.A., Castañer J. Voriconazole. Drugs Fut 1996;21:266-71.

9. Lewis J.S., Boucher H.W., Lubowski T.J., et al. Cost advantage of voriconazole over amphotericin B deoxycholate for primary treatment of invasive aspergillosis. Pharmacotherapy 2005;25:839-46.

10. Wenzel R., Del Favero A., Kibbler C., et al. Economic evaluation of voriconazole compared with conventional amphotericin B for the primary treatment of aspergillosis in immunocompromised patients. J Antimicrob Chemother 2005;55:352-61.

11. Donnelly J.P., De Pauw B.E. Voriconazole - a new therapeutic agent with an extended spectrum of antifungal activity. Clin Microbiol Infect 2004;10:107-17.

12. Package insert. Vfend ${ }^{\circledR}$ (voriconazole). Pfizer Laboratories.

13. Hoppe-Tichy T., Wenzel S., Gehrig A.K., Nguyen H. Stability of voriconazole in infusion bags. Pharmazie 2005;60:77-8.

14. Sahraoui L., Chiadmi F., Schlatter J., et al. Stability of voriconazole injection in $0.9 \%$ sodium chloride and $5 \%$ dextrose injections. Am J Health-Syst Pharm 2006;63:1423-26.

15. Cadrobbi J., Hecq J.P., Lebrun C., et al. Long-term stability of voriconazole $4 \mathrm{mg} / \mathrm{mL}$ in $5 \%$ dextrose polyvinyl chloride bags at $4{ }^{\circ} \mathrm{C}$. EJHP Science 2006;12:57-9.

16. Adams A.I.H., Bergold A.M. Development and validation of a high performance liquid chromatographic method for the determination of voriconazole content in tablets. Chromatographia 2005;62:429-34.

17. Watson D.G. Pharmaceutical Analysis. A Textbook for pharmacy students and pharmaceutical chemists. Edinburgh: Elsevier Churchill Livingstone, 2005.

18. CDER. Center for Drug Evaluation and Research - FDA. Reviewer guidance for validation of chromatographic methods 1994.

19. Moorhatch P., Chiou W.L. Interactions between drugs and plastic intravenous fluids bags. Part I: sorption studies on 17 drugs. Am J Hosp Pharm 1974;31:72-8.

20. Kowaluk E.A., Roberts M.S., Balckburn H.D., Polack A.E. Interactions between drugs and polyvinyl chloride infusion bags. Am J Hosp Pharm 1981;38:1308-14.

21. Illum L., Bundgaard H. Sorption of drugs by plastic infusion bags. Int J Pharm 1982;10:339-51.

22. Lackner T.E., Baldus D., Butler C.D., et al. Lidocaine stability in cardioplegic solution stored in glass bottles and polyvinyl chloride bags. Am J Hosp Pharm 1983;40:97-101.

23. Captisol@. www.captisol.com/Captisol/ProductApprovals.pdf. (accessed on 04/20/05). 